\title{
Amphiphilic semiconducting copolymer as compatibility layer for printing polyelectrolyte- gated OFETs
}

\author{
Hiam Sinno, Ha Tran Nguyen, Anders Hägerström, Mats Fahlman, Linda Lindell, \\ Olivier Coulembier, Philippe Dubois, Xavier Crispin, Isak Engquist and Magnus Berggren
}

\section{Linköping University Post Print}

N.B.: When citing this work, cite the original article.

Original Publication:

Hiam Sinno, Ha Tran Nguyen, Anders Hägerström, Mats Fahlman, Linda Lindell, Olivier Coulembier, Philippe Dubois, Xavier Crispin, Isak Engquist and Magnus Berggren, Amphiphilic semiconducting copolymer as compatibility layer for printing polyelectrolytegated OFETs, 2013, Organic electronics, (14), 3, 790-796.

http://dx.doi.org/10.1016/j.orgel.2012.12.031

Copyright: Elsevier

http://www.elsevier.com/

Postprint available at: Linköping University Electronic Press http://urn.kb.se/resolve?urn=urn:nbn:se:liu:diva-88980 


\title{
Amphiphilic semiconducting copolymer as compatibility layer for printing polyelectrolyte-gated OFETs
}

\author{
Hiam Sinno ${ }^{\mathrm{a}}$, Ha Tran Nguyen ${ }^{\mathrm{b}}$, Anders Hägerström ${ }^{\mathrm{a}}$, Mats Fahlman ${ }^{\mathrm{c}}$, Linda Lindell ${ }^{\mathrm{c}}$, Olivier \\ Coulembier $^{\mathrm{b}}$, Philippe Dubois ${ }^{\mathrm{b}}$, Xavier Crispin ${ }^{\mathrm{a}}$, Isak Engquist ${ }^{\mathrm{a}, *}$, Magnus Berggren $^{\mathrm{a}}$ \\ ${ }^{a}$ Department of Science and Technology, Organic Electronics, Linköping University, SE-601 74 Norrköping \\ (Sweden) \\ ${ }^{\mathrm{b}}$ Laboratory of Polymeric and Composite Materials, Center of Innovation and Research in Materials and \\ Polymers (CIRMAP), University of Mons-UMONS, Place du Parc 23, 7000 Mons (Belgium) \\ ${ }^{\mathrm{c}}$ Department of Physics, Chemistry and Biology, Linköping University, SE-581 83 Linköping (Sweden) \\ * Corresponding author. Tel.: +46 11 363401; fax: +46 11363270. \\ E-mail address: isak.engquist@liu.se (I. Engquist).
}

\begin{abstract}
We report a method for inkjet-printing an organic semiconductor layer on top of the electrolyte insulator layer in polyelectrolyte-gated OFETs by using a surface modification treatment to overcome the underlying wettability problem at this interface. The method includes depositing an amphiphilic diblock copolymer (P3HT- $b$-PDMAEMA). This material is designed to have one set of blocks that mimics the hydrophobic properties of the semiconductor (poly(3-hexylthiophene) or P3HT), while the other set of blocks include polar components that improve adhesion to the polyelectrolyte insulator. Contact angle measurements, atomic force microscopy, and x-ray photoelectron spectroscopy confirm formation of the desired surface modification film. Successful inkjet printing of a smooth semiconductor layer allows us to manufacture complete transistor structures that exhibit lowvoltage operation in the range of $1 \mathrm{~V}$.
\end{abstract}

Keywords: Block Copolymers, Inkjet Printing, Organic Field-Effect Transistors, Organic Electronics, Printed Electronics

\section{Introduction}

Printed organic electronics combines the flexibility of organic materials with the low cost, robustness, and high throughput of printing technologies.[1,2] In a majority of printed circuits, 
organic field effect transistors (OFETs) are essential components, and substantial research efforts have been dedicated to achieve transistors that combine printability with low-voltage operation. To this end, several groups have previously demonstrated electrolyte-gated OFETs (EGOFETs) fabricated using spin-coating and thermal evaporation techniques. The EGOFETs differ from conventional OFETs in the sense that an electrolyte is included as the gate insulator material instead of a dielectric layer.[3-5] Gating via an electrolyte enables the formation of electric double-layers (EDLs) at the gate/electrolyte and electrolyte/semiconductor interfaces upon gate biasing, induced by ion migration within the polyelectrolyte. The EDLs provide transistor configurations with high interfacial capacitances that make device operation at low voltages possible.[3-5] These qualities make EGOFETs suitable to manufacture using printing techniques since ultra-high resolution is typically difficult to achieve. However, several challenges have to be overcome when attempting to achieve a fully printed EGOFET. One of the main problems faced relates to adhesion properties at the semiconductor/electrolyte interface and is attributed to incompatible wetting characteristics. The common p-type semiconducting materials are typically hydrophobic, whereas the common polyelectrolyte materials are hydrophilic, which makes it difficult to print these two layers as a stack on top of each other. Thus, one of the main challenges in the production of EGOFETs is to find a suitable combination of materials and solvents having matched wettability characteristics but that do not dissolve each other.

Previous attempts towards all-printed EGOFETs include aerosol-jet-printed thin-film transistors gated ion-gels.[6] Such devices include a gelled electrolyte, based on an ionic liquid and a gelating block copolymer, as the gate insulator. This gel electrolyte is hydrophobic which makes deposition on top of the hydrophobic poly(3-hexylthiophene) P3HT semiconductor easy. However, one draw-back is that the anions from the gel can penetrate into the polymer bulk which leads to unwanted electrochemical reactions in the semiconductor.[7] 
Another approach to achieve working electrolyte-gated transistors is the all printed hygroscopic insulator field effect transistor (HIFET)[8] where reverse gravure coating was used to apply the semiconductor and insulator.[9] This transistor also relies on oxidation/reduction of the semiconductor by ions from the electrolyte. Printed electrolytes have also been used in electrochemical transistors and can be fabricated both by inkjet[10] and screen printing[11]. The aim of this work is to demonstrate a method to print the organic semiconductor on top of the polyelectrolyte to enable all-printed processing of EGOFETs.

In this work, we are addressing the most crucial fabrication step to manufacture EGOFETs, which is the semiconductor/electrolyte interface, where wettability incompatibilities have to be overcome and where a smooth and homogeneous interface is crucial for the performance of the transistor. We have investigated a surface modification treatment of the electrolyte film by the aid of a diblock copolymer (BC) to render the film relatively more hydrophobic, thereby enhancing the printability of the hydrophobic semiconductor layer on top. A BC derivative has been synthesized composed of two blocks that chemically mimic the properties of the semiconductor and the electrolyte, respectively. With this material we aim to promote the printability of the semiconductor without interfering electrically with the functionality of the transistor, since the hydrophobic semiconducting block in the copolymer is expected to be part of the semiconducting channel of the transistor. In contrast with typical insulating amphiphilic interfacial agents, the presence of a semiconducting block ensures close distance between the electronic charge carriers migrating along the semiconducting channel and the ionic charge carriers that form the EDLC at the semiconductor/electrolyte interface. A bottom-gate structure was implemented here because preliminary experiments show that it is not possible to do a top gate configuration using the $\mathrm{BC}$-treatment. We believe that the amine group in the $\mathrm{BC}$ is attracted to the negatively charged polyelectrolyte which leads to the formation of a layer of BC molecules on the polyelectrolyte surface with the polythiophene segment part facing 
outward towards the semiconducting layer. This driving force for $\mathrm{BC}$ layer formation is not present in the top-gate structure where the $\mathrm{BC}$ would be deposited on the semiconductor. Thus, our BC-treatment is suitable for use only when the semiconducting layer is printed on top of the electrolyte layer. Inkjet printing was selected as the deposition method because of its flexibility and short lead-time for small sample series, and because wettability incompatibility is a well-known issue for this printing technique.[12-15] Also, with inkjet printing we can deliver a wide range of functional materials while minimizing material waste.

The block copolymer P3HT- $b$-PDMAEMA (Fig. 1) consists of a poly(3-hexylthiophene) block (molar mass, $M_{n} \sim 7,000 \mathrm{~g} \mathrm{~mol}^{-1}$ ) covalently linked to a poly $(N, N$-dimethylaminoethyl methacrylate) segment $\left(M_{n} \sim 16,500 \mathrm{~g} \mathrm{~mol}^{-1}\right)$. This combination of conjugated and nonconjugated segments results in a material that can phase-segregate into nanostructures due to the immiscibility of the covalently connected blocks. A detailed description of the synthesis of a polymer of this class is given in another paper.[16]

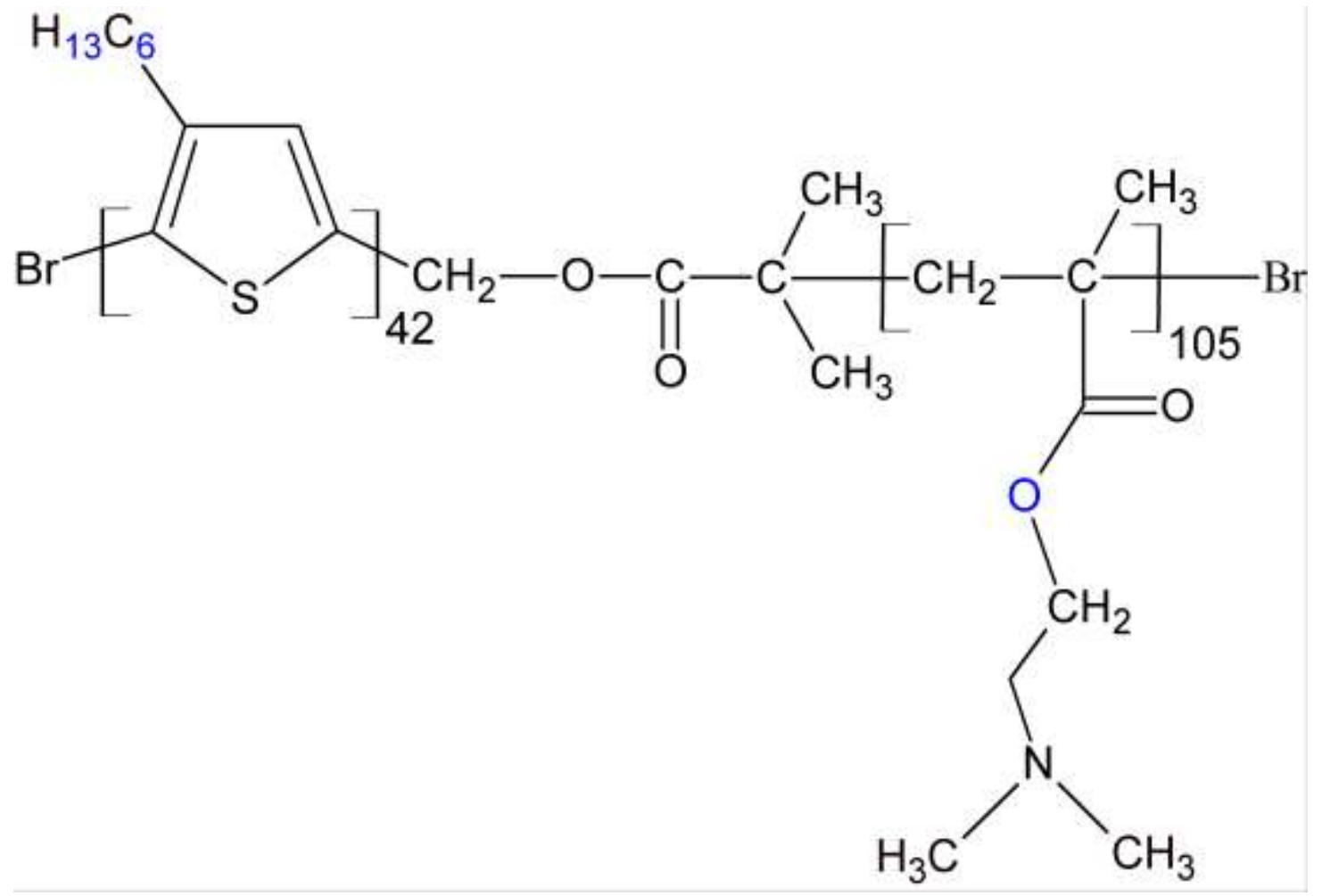

Figure 1. Chemical structure of the diblock copolymer P3HT-b-PDMAEMA. 


\section{Experimental}

2.1. Synthesis of poly(3-hexylthiophene)-block-poly(N,N-dimethylaminoethyl methacrylate) (P3HT- $b$-PDMAEMA):

The polymerization was performed in anhydrous THF by using P3HT-Br (or P3HT-MI), $\mathrm{CuBr}$, and HMTETA as macroinitiator, catalyst, and ligand, respectively. The initial molar ratio was $[\mathrm{M}]_{0}:[\mathrm{P} 3 \mathrm{HT}-\mathrm{MI}]_{0}:[\mathrm{CuBr}]_{0}:[\mathrm{HMTETA}]_{0}=100: 1: 1: 2$. A dry flask was charged with $\mathrm{CuBr}(4.1 \mathrm{mg}, 0.028 \mathrm{mmol}), \operatorname{HMTETA}(15.7 \mu \mathrm{l}, 0.057 \mathrm{mmol})$, THF $(1 \mathrm{ml})$, and a magnetic stirrer. The flask was fitted with a rubber septum and degassed with three successive vacuum nitrogen cycles. A second flask was charged with the P3HT-MI (200 mg, $\mathrm{M}_{\mathrm{n}}=7000 \mathrm{~g} \mathrm{~mol}^{-1}$, $0.02857 \mathrm{mmol})$, DMAEMA monomer $(0.5 \mathrm{ml}, 450 \mathrm{mg}, 2.857 \mathrm{mmol})$, and THF (2 ml). The flask was fitted with a rubber septum and degassed under nitrogen flow for a few minutes. The mixture was then transferred into the first flask and the polymerization was carried out under stirring at $60{ }^{\circ} \mathrm{C}$ for 16 hours. The reaction was stopped by cooling down the reaction medium in liquid nitrogen. Then, THF was added to dilute the solution. Copper catalyst was removed by filtration of the THF solution through an alumina oxide column. The diblock copolymers were recovered by precipitation from cold heptane, filtration and drying up to constant weight. ${ }^{1} \mathrm{H}$ NMR (300 MHz, $\left.\mathrm{CDCl}_{3}\right) ; \delta(\mathrm{ppm}): 6.96(\mathrm{~s}, 1 \mathrm{H}), 5.29(\mathrm{~s}, 2 \mathrm{H}), 4.06(\mathrm{~s}, 2 \mathrm{H}), 2.78(\mathrm{t}, 2 \mathrm{H})$, 2.57 (s, 2H), 2.28 (s, 6H), 1.90 (s, 2H), 1.69 (sex, 2H), 1.49 (q, 6H), 1.04 (s, 9H), 0.89 (t, 3H). $\mathrm{M}_{\mathrm{n}} \mathrm{GPC} \sim 19300 \mathrm{~g} \mathrm{~mol}^{-1} . \mathrm{\oplus}, 1.7 . \mathrm{M}_{\mathrm{n}} \mathrm{NMR} \sim 23,500 \mathrm{~g} \mathrm{~mol}^{-1}$.

\subsection{Device fabrication:}

The p-type EGOFET was manufactured by thermally evaporating (50 nm thick) gold ( $\mathrm{Au})$ on top of (5 nm thick) chromium ( $\mathrm{Cr}$ ) (bottom gate electrode) through a shadow mask on a $\mathrm{Si} / \mathrm{SiO}_{2}$ wafer. A (100 nm thick) polyelectrolyte film consisting of a mixture of 1:14 vol:vol poly(styrene sulfonic acid) (PSSH, Sigma-Aldrich with a molecular weight of about 75000 and a concentration of $18 \mathrm{wt}$. \% in de-ionized water) to de-ionized water with $0.3 \%$ zonyl as a 
surfactant was spincoated on top of the gate electrode and annealed under vacuum at $110{ }^{\circ} \mathrm{C}$ for $90 \mathrm{~s}$. Then, a PSSH film treatment was performed by dipping the sample in a solution consisting of (0.01 mg ml-1 P3HT- $b$-PDMAEMA in toluene) for 1 minute. The samples were ultrasonicated in toluene for 5 minutes to remove excess layers of the $\mathrm{BC}$ and to break any $\mathrm{BC}$ micelles in order to achieve good contact with the PSSH surface. The organic semiconductor solution consisting of $4 \mathrm{mg} \mathrm{ml}^{-1}$ regioregular poly(3-hexylthiophene) (rr-P3HT, Sigma-Aldrich with a regioregularity greater than $95 \%$ and a molecular weight of $15000-45000$ ) in $68 \%$ ortho-dichlorobenzene (oDCB) and 32\% mesitylene was printed using the Dimatix material printer DMP-2800 forming a relatively continuous film (40 $\mathrm{nm}$ thick). The semiconductor solvent formulation and the printer platen temperature control the wetting and drying behavior of the printed semiconductor film on the PSSH electrolyte layer. A pure oDCB formulation results in poor spreading and wettability of the printed film because of its high surface tension and high boiling point; therefore, mesitylene was added to the solvent formulation since it has a lower boiling point and lower surface tension. The ratio of solvents was chosen according to a previous reported study that shows this formulation to provide an optimum wettability of the printed film on top of PEDOT:PSS.[17] Cartridges with a $10 \mathrm{pl}$ drop volume were used, the drop spacing was set to $40 \mu \mathrm{m}$, a single nozzle was used for jetting the P3HT solution with a maximum jetting frequency of $10 \mathrm{kHz}$, and the printer platen temperature was set to $30{ }^{\circ} \mathrm{C}$. The rr-P3HT film was annealed under nitrogen at $120{ }^{\circ} \mathrm{C}$ for 10 minutes. Finally, $(100 \mathrm{~nm}$ thick) Au was thermally evaporated through a shadow mask forming the source and drain top contacts. The channel length and width of the transistor were $10 \mu \mathrm{m}$ and $700 \mu \mathrm{m}$ respectively. The structure of our device is shown in Fig. 2. For comparison reasons, a sample with a spincoated PSSH film was treated with a common surfactant (dodecyltrimethylammonium chloride in diethylcarbonate) by dipping it in this solution for 1 minute and then rinsing it in toluene where the sample was ultrasonicated for 5 minutes to get rid of excess layers of the 
surfactant. Then, a rr-P3HT layer was inkjet-printed on top of the treated PSSH layer using the same parameters indicated above.

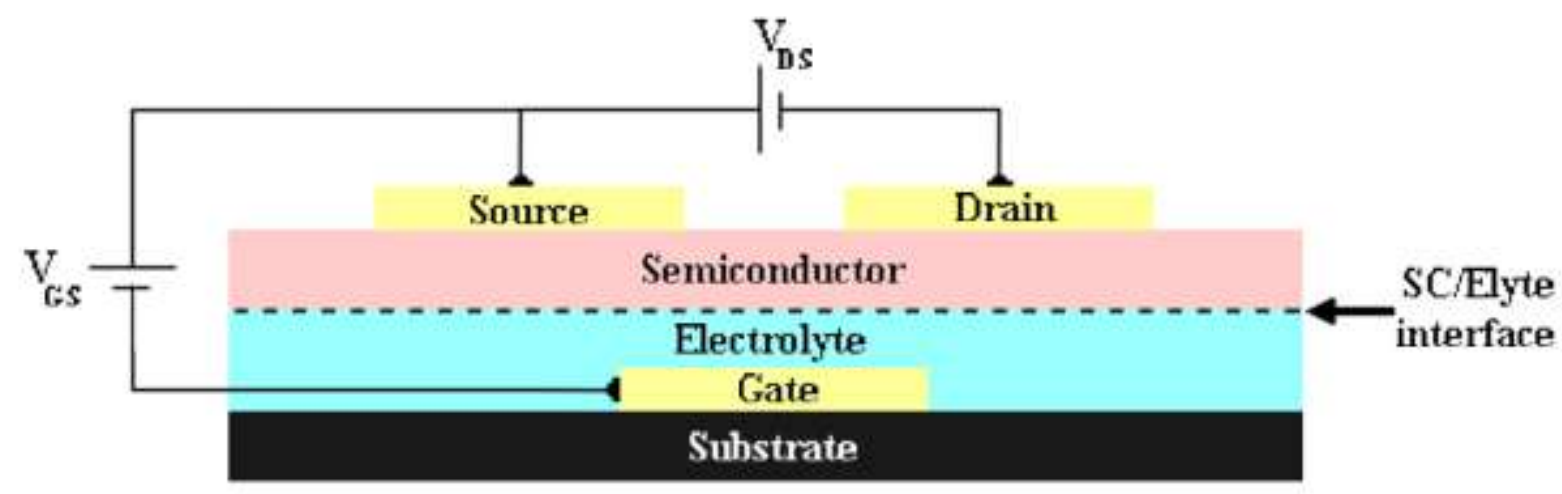

Figure 2. EGOFET design structure. The critical semiconductor/electrolyte is marked with a dotted line. The external circuit used for transistor measurements is also indicated.

\subsection{Device characterization:}

Contact angle measurements were recorded using a goniometer (CAM 200, KSV). The AFM images were obtained using a Veeco Dimension 3100 atomic force microscopy (AFM). X-ray photoelectron spectroscopy (XPS) measurements were performed using a Scienta ESCA 200 instrument. The transistor characteristics reported here were measured with a Keithley 4200-SCS parameter analyzer. All electrical measurements were made in ambient air (relative humidity ca. $40 \%$ ) at room temperature.

\section{Results and discussion}

The block copolymer treatment was performed by dipping the PSSH-coated sample in a solution of P3HT- $b$-PDMAEMA dissolved in toluene. It is important to use a non-polar solvent such as toluene in order to avoid dissolving the underlying PSSH layer. To some extent, this will result in the formation of micelles in the solution, as evidenced by the previously reported differences in the absorption spectra of the $\mathrm{BC}$ in polar and non-polar solvents, respectively.[16] We expect these micelles to break in contact with the polar PSSH surface and contribute to the formation of a BC film; however, it will be seen below that a few 
defects remain which we believe can be removed by further optimization of the solvent formulation.

Contact angle measurements of hexadecane and DI-water on the spin-coated PSSH film prior to and after the BC-treatment have been recorded using a goniometer, with results shown in Table 1. Various BC-treatment times were investigated to find the shortest treatment that would yield the sought-after wettability improvement. The contact angles change considerably after the BC-treatment indicating a less hydrophilic surface, which should promote printability of the rr-P3HT solution on top of the electrolyte. We can see that longer treatment times results in lower hexadecane contact angles, but that already 1 minute treatment causes a significant drop compared to the untreated surface. We have proceeded with the short 1 minute $\mathrm{BC}$ treatment time since the change in the contact angle was sufficient to achieve printability and since the thinnest possible $\mathrm{BC}$ layer is desirable in order to avoid degradation of transistor performance. The contact angle measurements were conducted on 10 different samples for each treatment, with mean values and standard deviation shown in Table 1. The low standard deviation values indicate that the treatment is repeatable.

The surface tension or surface free energy (SFE) of both samples was calculated using the Owens-Wendt theoretical model.[18] The model estimates the long-range dispersion (Lifshitz-Van derWaals, $\gamma^{\mathrm{d}}$ ) and the short-range polar (hydrogen bonding, $\gamma^{\mathrm{p}}$ ) components of SFE according to the following equation:

$$
(1+\cos \theta) \gamma_{1}=2\left(\gamma_{s}^{d}\right)^{1 / 2}\left(\gamma_{1}^{d}\right)^{1 / 2}+2\left(\gamma_{s}^{p}\right)^{1 / 2}\left(\gamma_{1}^{p}\right)^{1 / 2}
$$

where $\gamma_{\mathrm{s}}$ and $\gamma_{1}$ are the SFE of the surface and the liquid respectively, and $\theta$ is the contact angle. The overall surface tension $\gamma$ is a combination of dispersive $\gamma^{\mathrm{d}}$ and polar $\gamma^{\mathrm{p}}$ components. Substituting both contact angle and surface tension values of DI-water and hexadecane (at 21 ${ }^{\circ} \mathrm{C}$, DI water: $\gamma=72.8 \mathrm{mN} \mathrm{m}^{-1}, \gamma^{\mathrm{d}}=21.8 \mathrm{mN} \mathrm{m}^{-1}, \gamma^{\mathrm{p}}=51 \mathrm{mN} \mathrm{m}^{-1}$; Hexadecane: $\gamma=27.47 \mathrm{mN}$ 
$\left.\mathrm{m}^{-1}, \gamma^{\mathrm{d}}=27.47 \mathrm{mN} \mathrm{m}^{-1}, \gamma^{\mathrm{p}}=0 \mathrm{mN} \mathrm{m}^{-1}\right)[19]$ in Equation 1, the samples surface tension were calculated and are shown in Table $\mathbf{1 .}$

Table 1. Contact angle data for pristine rr-P3HT and PSSH, and for BC-treated surfaces, and calculated surface tension $(\gamma)$, dispersive $\left(\gamma^{\mathrm{d}}\right)$ and polar $\left(\gamma^{\mathrm{p}}\right)$ components of the surface tension of all samples at $20{ }^{\circ} \mathrm{C}$. The contact angle measurements were conducted on 10 samples for each treatment and the mean values and standard deviations are given.

\begin{tabular}{lcc}
\hline & Contact angles $\left[^{\circ}\right]$ & \\
\hline Samples & Dl water & Hexadecane \\
\hline Pristine rr-P3HT & $101.63 \pm 0.26$ & $32.78 \pm 0.42$ \\
Pristine PSSH & $18.74 \pm 0.35$ & $50.54 \pm 0.86$ \\
BC-treated PSSH & & $39.01 \pm 0.14$ \\
$\mathbf{1}$ minute & $44.49 \pm 0.15$ & $38.66 \pm 0.18$ \\
$\mathbf{5}$ minutes & $46.66 \pm 0.11$ & $35.65 \pm 0.15$ \\
$\mathbf{3 0}$ minutes & $49.60 \pm 0.14$ & $31.59 \pm 0.19$ \\
$\mathbf{1}$ hour & $52.42 \pm 0.18$ & $26.14 \pm 0.46$ \\
\hline
\end{tabular}

Surface tension $\left(\gamma, \mathrm{mN} \mathrm{m}^{-1}\right)$ at $20^{\circ} \mathrm{C}$

\begin{tabular}{llll}
\hline Samples & $\gamma$ & $\gamma^{\mathrm{d}}$ & $\gamma^{\mathrm{p}}$ \\
\hline Pristine rr-P3HT & $24.1 \pm 0.05$ & $23.26 \pm 0.10$ & $0.83 \pm 0.05$ \\
Pristine PSSH & $69.08 \pm 0.16$ & $18.36 \pm 0.26$ & $50.71 \pm 0.42$ \\
BC-treated PSSH & & & \\
$\mathbf{1}$ minute & $54.04 \pm 0.10$ & $21.69 \pm 0.03$ & $32.35 \pm 0.13$ \\
$\mathbf{5}$ minutes & $52.51 \pm 0.06$ & $21.78 \pm 0.04$ & $30.73 \pm 0.11$ \\
$\mathbf{3 0}$ minutes & $50.59 \pm 0.09$ & $22.56 \pm 0.03$ & $28.03 \pm 0.12$ \\
$\mathbf{1}$ hour & $48.87 \pm 0.12$ & $23.55 \pm 0.04$ & $25.32 \pm 0.16$ \\
$\mathbf{1 0}$ hours & $44.44 \pm 0.17$ & $24.73 \pm 0.09$ & $19.71 \pm 0.26$ \\
\hline
\end{tabular}

It can be seen from both contact angle and surface tension data presented in Table 1 that the electrolyte layer is rendered more hydrophobic after the BC-treatment thus enhancing the printability of the rr-P3HT semiconductor layer on top. We conclude that although a dilute BC solution was used, and the treatment time was short (1 minute), the desired surface modification has taken place. Obviously, a fully P3HT-like surface has not been achieved, but the aim here is to obtain a surface where rr-P3HT can be inkjet-printed using an interlayer that is as thin and smooth as possible. 
Atomic force microscopy (AFM) measurements of the PSSH electrolyte surface were performed before and after the BC-treatment. Results are shown in Fig. 3. We observe that the roughness of the BC-treated PSSH surface is larger and that the film exhibits more texture than the untreated surface. RMS roughness values are $0.14 \mathrm{~nm}$ for the untreated PSSH surface and $0.7 \mathrm{~nm}$ for the BC-treated PSSH surface. These results indicate that the BC-treatment results in the formation of a layer of the diblock copolymer on top of the PSSH surface with a different surface morphology still smooth to provide a good template for the following process steps. In Fig. 3b, we note a few elevated areas spread over the surface that are about 3 to $4 \mathrm{~nm}$ high and may consist of unbroken micelles. However, given their small height and area, they should be of little significance for the following printing of P3HT.

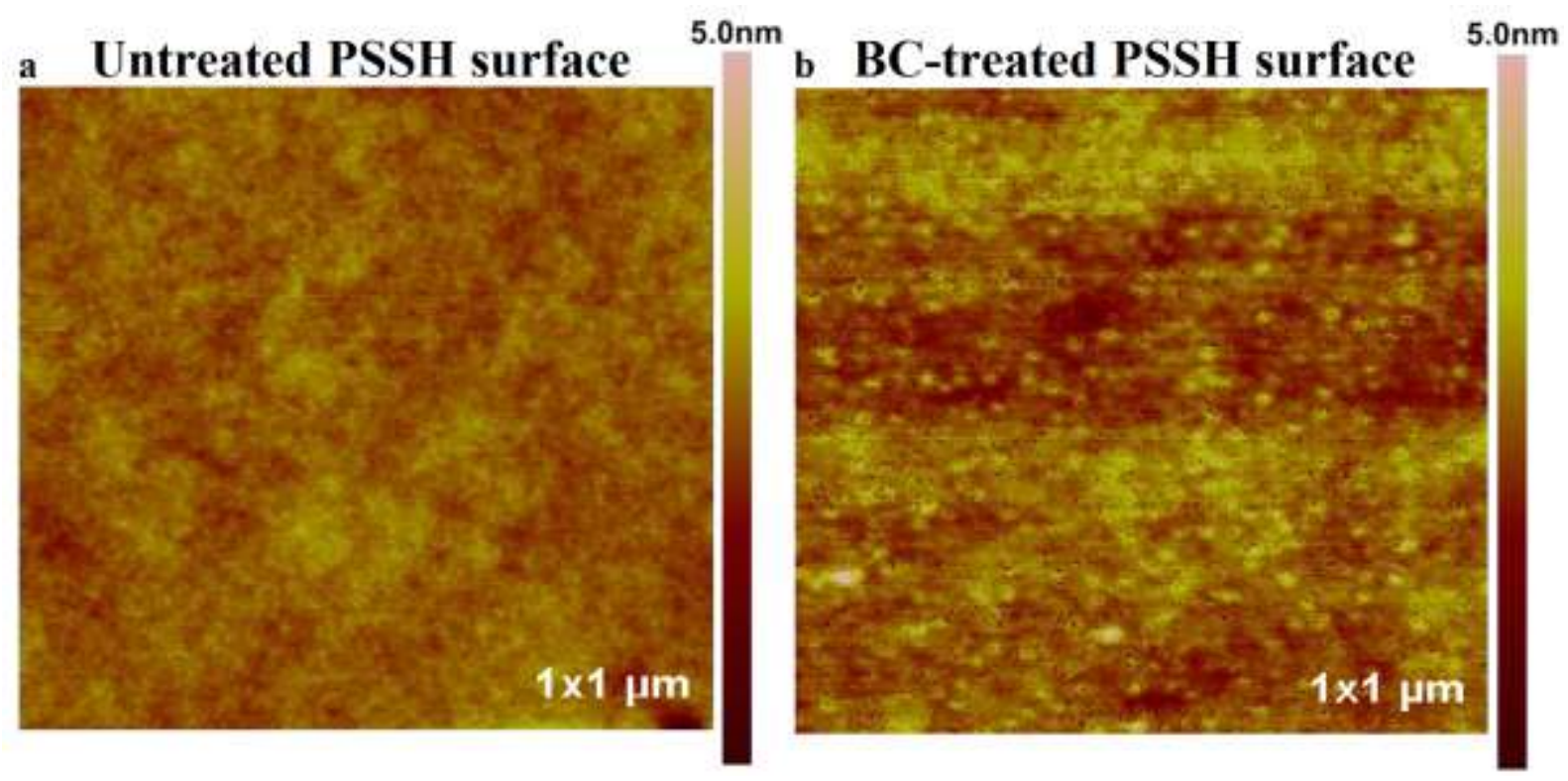

Figure 3. AFM height images of the (a) untreated and (b) BC treated PSSH electrolyte surfaces.

The BC-treatment results were also investigated by XPS. Measurements were performed on an untreated PSSH film coated on a Si substrate and also on a BC-treated PSSH film according to the standard process described in the experimental section. Normal emission (flat) and $67^{\circ}$ glancing angle emission (tilted) measurement geometries were used for both samples. Of 
special interest are the $S(2 p)$ peaks, shown in Fig. 4 for the untreated and the BC-treated samples. The untreated PSSH surface (Fig. 4a) gives rise to a high binding energy double peak at $(168-172 \mathrm{eV})$, originating from the sulfonate group due to the presence of three electronegative oxygen atoms in the surrounding.[20] In the $S(2 p)$ narrow scan of the BCtreated PSSH surface (Fig. 4b), the high binding energy peak originates from the PSSH layer while the low binding energy peak $(164-167 \mathrm{eV})$ originates from the thiophene part of the BC film. The existence of the thiophene peak proves that the BC-treatment was successful. The intensity of the thiophene peak is quite low which indicates that the copolymer layer is very thin. The increase in the thiophene/sulfonate signal ratio when the sample is tilted ("flat" versus "tilted" in the $164-167 \mathrm{eV}$ region of Fig. 4b) indicates that the copolymer film is at the surface of the PSSH layer, which is in agreement with the contact angle and surface tension data.
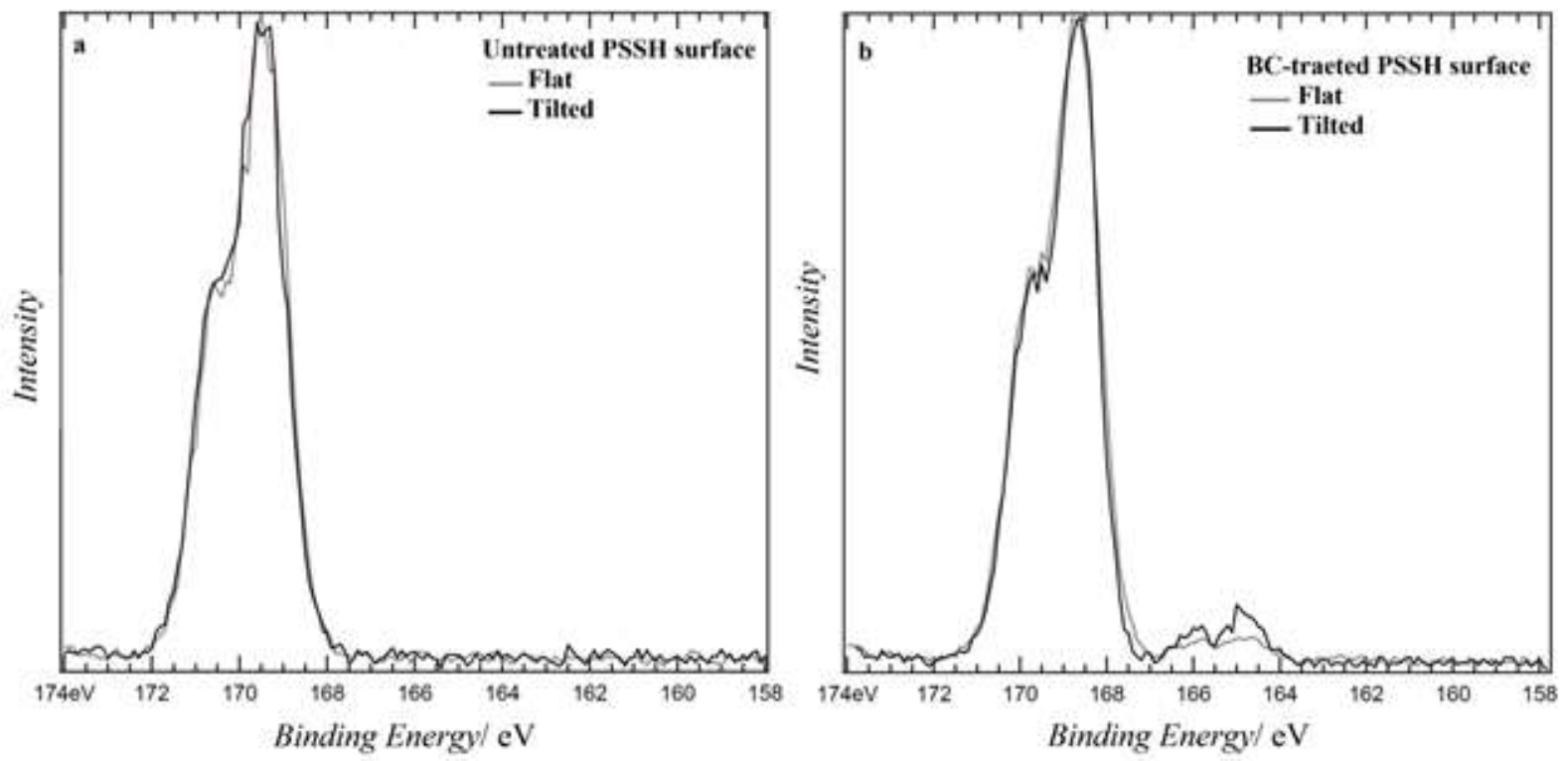

Figure 4. (a) XPS narrow-scan of $S(2 p)$ peak for the untreated PSSH surface (flat and tilted emission), (b) XPS narrow-scan of S(2p) peak for BC treated PSSH surface (flat and tilted emission).

In Fig. 5, the difference between an inkjet printed rr-P3HT layer on top of untreated and 1minute BC-treated PSSH films is shown (Fig. 5 a and b). The printed P3HT film on top the 
untreated PSSH layer results in the formation of de-wetted lines corresponding to the passage of the inkjet printer head. It is clear that the resulting printed rr-P3HT film on top of the untreated PSSH film is inhomogeneous and has many dewetting spots while the printed rrP3HT film on top of the BC-treated PSSH film completely wets the electrolyte film and is essentially homogeneous. This is direct proof that the BC-treatment provides the desired global surface modification effect that enables printing fabrication of the polymer semiconductor on top of the polyelectrolyte insulator. For the purpose of comparison, a PSSH film was also treated with a common surfactant (dodecyltrimethylammonium chloride in diethylcarbonate), but attempts to inkjet-print the rr-P3HT layer on top resulted in an inhomogeneous film with a high density of dewetting spots, similar to the results for the untreated PSSH (Fig. 5c). Additionally, an image of the 1-minute BC-treated PSSH electrolyte film is shown in Fig. 5d. We can see that the BC-treatment results in the formation of a few "white dots" spread over the BC film, possibly consisting of aggregates of unbroken micelles as mentioned above. After printing the semiconductor layer, these white dots appear as defects in the form of black spots as seen in Fig. 5b. We expect that further optimization of the BC-treatment will remove the micelles and thereby also these defects. Summarizing our measurements, the diblock copolymer treatment of the PSSH electrolyte layer has been shown to enhance the printability of the rr-P3HT semiconducting layer to a point where a continuous film is obtained, which holds promise for the fabrication of a working transistor. 
a

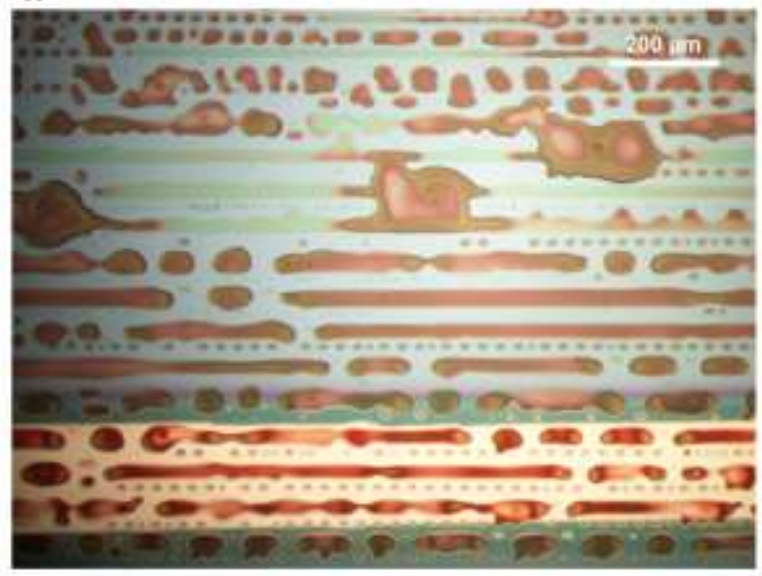

c

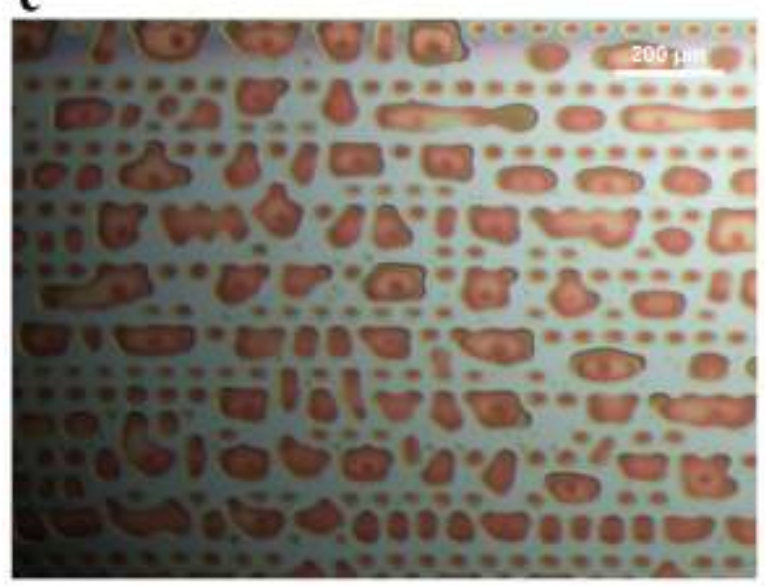

b

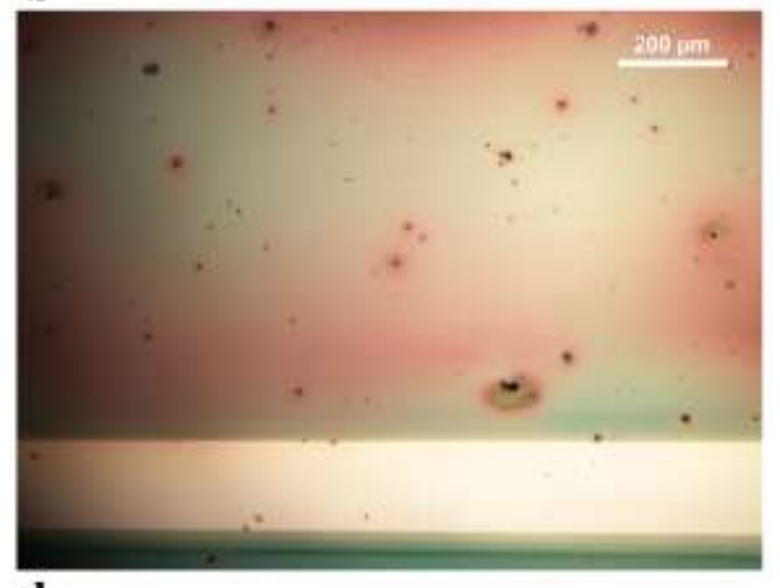

d

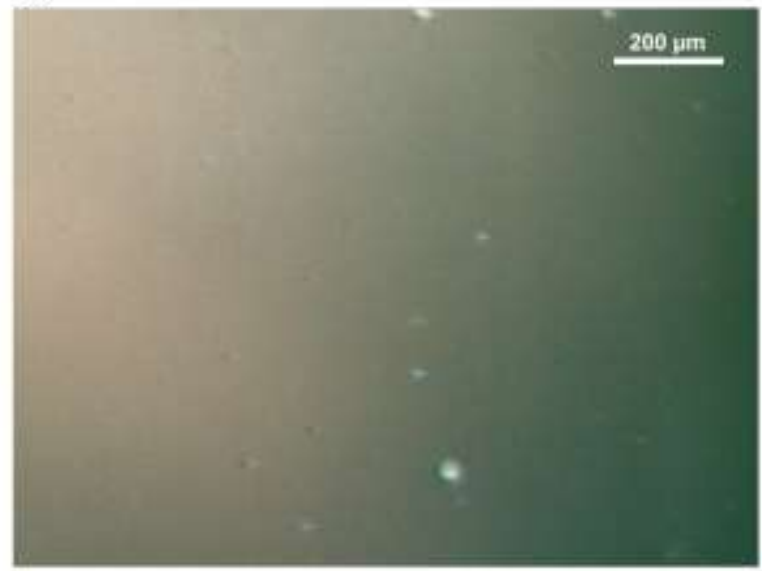

Figure 5. Optical microscopy images of (a) an inkjet-printed rr-P3HT film on top of untreated PSSH electrolyte film, (b) inkjet-printed rr-P3HT film on top of 1-minute BC-treated PSSH electrolyte film, (c) inkjet-printed rrP3HT film on top of dodecyltrimethylammonium-chloride-in-diethylcarbonate-treated PSSH electrolyte film, (d) 1 minute BC-treated PSSH electrolyte film. The formation of the lines in the untreated and surfactant treated images (a and c) corresponds to the passage of the printer head. The gold-colored stripe at the bottom of images (a and $\mathrm{b}$ ) is the bottom Au gate.

As a final step, full EGOFETs were fabricated for electrical characterization. This was obviously only possible on BC-treated samples. The resulting EGOFET transistors (10 samples) were electrically characterized. Transfer and output curves of one of the fabricated transistors are shown in Fig. 6a and Fig. 6b, respectively. The obtained on/off current ratio $I_{\text {on }} / I_{\text {off }}=40 \pm 6$. This is lower, but in the same order of magnitude, as compared to an identical EGOFET manufactured using spin-coating without BC-treatment $\left(\mathrm{I}_{\mathrm{on}} / \mathrm{I}_{\mathrm{off}} \approx 100\right)$. The leakage current (gate current) is typically less than one order of magnitude lower than $I_{D}$ at a given voltage. The threshold voltage is found to be $-0.63 \pm 0.05 \mathrm{~V}$ which is higher than the typical value observed in identical transistors manufactured with spin-coating $\left(\mathrm{V}_{\mathrm{T}}=-0.45 \mathrm{~V}\right)$. The 
field effect mobility at saturation is estimated to be $0.15 \pm 0.004 \mathrm{~cm}^{2} \mathrm{~V}^{-1} \mathrm{~s}^{-1}$ (calculated for $\mathrm{C}_{\mathrm{i}}=$ $3 \mu \mathrm{F} \mathrm{cm}{ }^{-2}$, at $\mathrm{V}_{\mathrm{D}}=-1 \mathrm{~V}$ and $\mathrm{V}_{\mathrm{G}}=-1.5 \mathrm{~V}$ ). The EGOFET shows a minor hysteresis behavior between the upward and downward scans for the transfer curve which we attribute to the fact that the accumulation of charges in the channel takes a few seconds (sweep rate of the upward scan is around $10 \mathrm{~s}$ ), and thus at the end of the downward scan of the transfer curve, the density of accumulated charges is higher which leads to a higher drain current. Overall, the transistor performance is not impressive and the on/off ratio is lower compared to conventional EGOFETs that are fabricated using spin-coating, vacuum evaporation, and photolithography patterning techniques. We believe this to be partly due to the use of the bottom-gate structure (for reasons stated above) which exposes the semiconductor to the ambient atmosphere. Also, the presence of the $\mathrm{BC}$ layer adds a thin interface to the critical zone where field effect takes place. However, it is clear that a working transistor can be built and we are confident that improved processing and optimized BC-treatment can improve the performance. Thus, the present measurements demonstrate the potential of the BC-treatment as a route towards low cost all-printed circuit applications.

\section{Conclusions}

In conclusion, we have shown that by applying a surface modification treatment to the hydrophilic electrolyte film using the P3HT- $b$-PDMAEMA diblock copolymer we can render the film more hydrophobic. This enables inkjet-printing of the hydrophobic rr-P3HT semiconductor layer on top of the electrolyte layer while minimizing the electrical influence on the functionality of the transistor, since the composition of the $\mathrm{BC}$ matches the materials surrounding it. This approach enables the production of low-voltage EGOFETs with inkjetprinted rr-P3HT semiconductor layer providing a route towards all-inkjet printed electronics. 

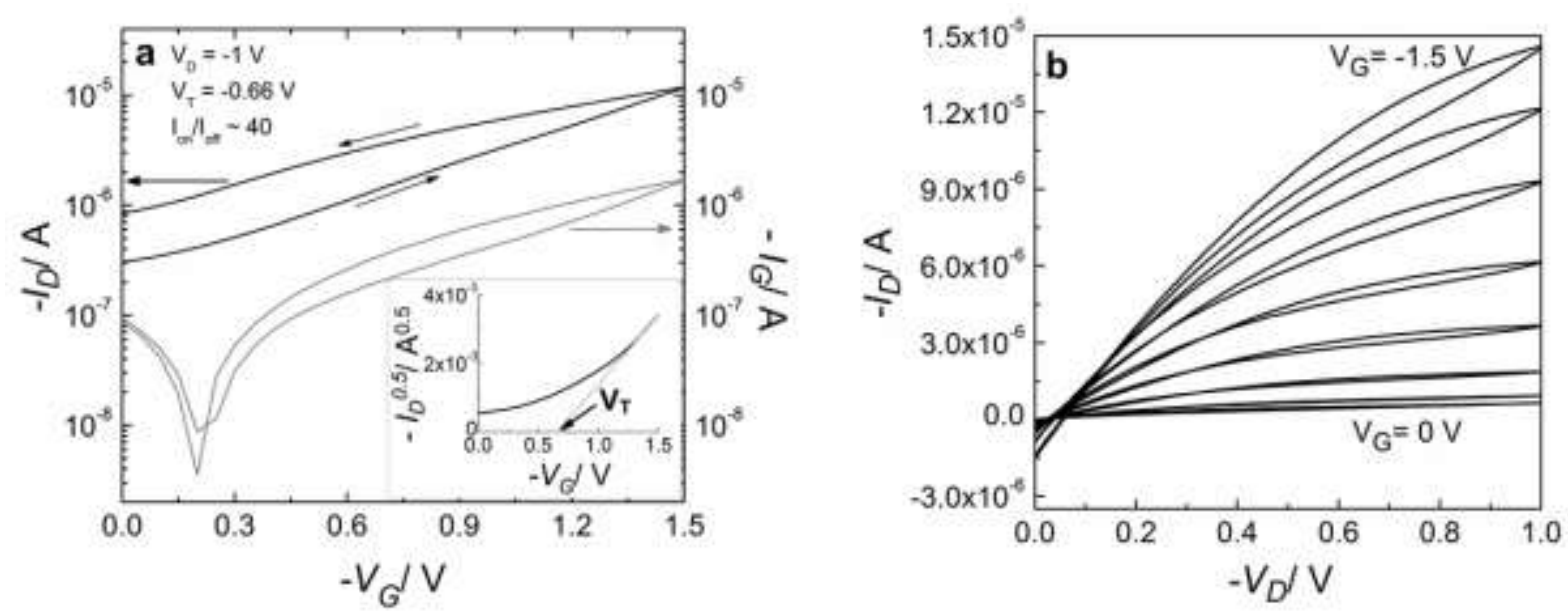

Figure 6. Current-voltage characteristics of a p-type EGOFET with inkjet-printed rr-P3HT layer having $10 \mu \mathrm{m}$ channel length and $700 \mu \mathrm{m}$ channel width. (a) Transfer curve with an inset showing the threshold voltage extraction from the linear fit to the square root of the drain current, (b) Output curves measured at the following $\mathrm{V}_{\mathrm{G}}$ values $0,-0.21 \mathrm{~V},-0.42 \mathrm{~V},-0.64 \mathrm{~V},-0.85 \mathrm{~V},-1.07 \mathrm{~V},-1.28 \mathrm{~V},-1.5 \mathrm{~V}$.

\section{Acknowledgements}

This work has been supported by Acreo, PEA-VINNOVA and PEA-PPP-TVV, OPEN-SSF, Önnesjö, KAW, the European Commission and Région Wallonne FEDER program (MateriaNova) and OPTI'MAT program of excellence, the Interuniversity Attraction Pole program of the Belgian Federal Science Policy Office (PAI 6/27) and by FNRS-FRFC. O.C. is FNRS Research Associate.

\section{References}

[1] H. Ma, H.-L. Yip, F. Huang, A.K.Y. Jen, Interface Engineering for Organic Electronics, Adv. Funct. Mater., 20 (2010) 1371-1388.

[2] M. Berggren, D. Nilsson, N.D. Robinson, Organic materials for printed electronics, Nat. Mater. , 6 (2007) 3-5.

[3] L. Herlogsson, X. Crispin, N.D. Robinson, M. Sandberg, O.J. Hagel, G. Gustafsson, M. Berggren, Low-Voltage Polymer Field-Effect Transistors Gated via a Proton Conductor, Adv. Mater., 19 (2007) 97-101.

[4] O. Larsson, E. Said, M. Berggren, X. Crispin, Insulator Polarization Mechanisms in Polyelectrolyte-Gated Organic Field-Effect Transistors, Adv. Funct. Mater., 19 (2009) 33343341.

[5] E. Said, X. Crispin, L. Herlogsson, S. Elhag, N.D. Robinson, M. Berggren, Polymer fieldeffect transistor gated via a poly(styrenesulfonic acid) thin film, Appl. Phys. Lett., 89 (2006) 143507-143510.

[6] Y. Xia, W. Zhang, M. Ha, J.H. Cho, M.J. Renn, C.H. Kim, C.D. Frisbie, Printed Sub-2 V Gel-Electrolyte-Gated Polymer Transistors and Circuits, Adv. Funct. Mater., 20 (2010) 587 594.

[7] J.H. Cho, J. Lee, Y. Xia, B. Kim, Y. He, M.J. Renn, T.P. Lodge, D. Frisbie, Printable iongel gate dielectrics for low-voltage polymer thin-film transistors on plastic, Nat. Mater., 7 (2008) 900-906.

[8] T.G. Bäcklund, H.G.O. Sandberg, R. Österbacka, H. Stubb, Current modulation of a hygroscopic insulator organic field-effect transistor, Appl. Phys. Lett., 85 (2004) 3887-3889. 
[9] D. Tobjö̊rk, N.J. Kaihovirta, T. Mäkelä, F.S. Pettersson, R. Österbacka, All-printed lowvoltage organic transistors, Org. Electron., 9 (2008) 931-935.

[10] L. Basiricò, P. Cosseddu, A. Scidà , B. Fraboni, G.G. Malliaras, A. Bonfiglio, Electrical characteristics of ink-jet printed, all-polymer electrochemical transistors, Org. Electron., 13 (2012) 244-248.

[11] T. Blaudeck, P.A. Ersman, M. Sandberg, S. Heinz, A. Laiho, J. Liu, I. Engquist, M.

Berggren, R.R. Baumann, Simplified Large-Area Manufacturing of Organic Electrochemical Transistors Combining Printing and a Self-Aligning Laser Ablation Step, Adv. Funct. Mater., (2012).

[12] P. Calvert, Inkjet Printing for Materials and Devices, Chem. Mater., 13 (2001) 32993305.

[13] J.Z. Wang, Z.H. Zheng, H.W. Li, W.T.S. Huck, H. Sirringhaus, Dewetting of conducting polymer inkjet droplets on patterned surfaces, Nat. Mater., 3 (2004) 171-176.

[14] F. Varela, E. Armendariz, C. Wolluschek, Inkjet printed electronics: The wet on wet approach, Chem. Eng. Process., 50 (2011) 589-591.

[15] M. Plötner, T. Wegener, S. Richter, S. Howitz, W.J. Fischer, Investigation of ink-jet printing of poly-3-octylthiophene for organic field-effect transistors from different solutions, Synth. Met., 147 (2004) 299-303.

[16] H. Nguyen, O. Coulembier, J. De Winter, P. Gerbaux, X. Crispin, P. Dubois, Novel regioregular poly(3-hexylthiophene)-based polycationic block copolymers, Polym. Bull., 66 (2011) 51-64.

[17] C.N. Hoth, S.A. Choulis, P. Schilinsky, C.J. Brabec, High Photovoltaic Performance of Inkjet Printed Polymer:Fullerene Blends, Adv. Mater., 19 (2007) 3973-3978.

[18] G. Lamour, A. Eftekhari-Bafrooei, E. Borguet, S. Souès, A. Hamraoui, Neuronal adhesion and differentiation driven by nanoscale surface free-energy gradients, Biomaterials, 31 (2010) 3762-3771.

[19] D.Y. Kwok, D. Li, A.W. Neumann, Evaluation of the Lifshitz-van der Waals/Acid-Base Approach To Determine Interfacial Tensions, Langmuir, 10 (1994) 1323-1328.

[20] X. Crispin, S. Marciniak, W. Osikowicz, G. Zotti, A.W.D. van der Gon, F. Louwet, M. Fahlman, L. Groenendaal, F. De Schryver, W.R. Salaneck, Conductivity, morphology, interfacial chemistry, and stability of poly(3,4-ethylene dioxythiophene)-poly(styrene sulfonate): A photoelectron spectroscopy study, J. Polym. Sci., Part B: Polym. Phys. , 41 (2003) 2561-2583. 